Supporting information

\title{
Hybrids of Upconversion Nanoparticles and Silver Nanoclusters Ensure Superior Bactericidal Capability via Combined Sterilization
}

Xun Liü, Zihan Cheng", Hui Wen, Shangqing Zhang, Mingli Chen*, Jianhua Wang*

Research Center for Analytical Sciences, Department of Chemistry, College of Sciences, Northeastern University, Shenyang, China, 110819

*E-mail: chenml@mail.neu.edu.cn(M.-L.Chen); jianhuajrz@mail.neu.edu.cn (J.H.Wang).

Tel: +862483688944 


\section{Table of contents}

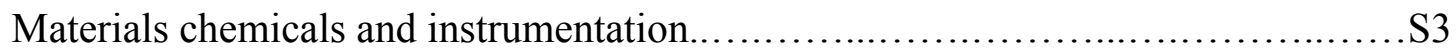

Figure S1 Figure S1. XRD pattern of UCNPs, UCNPs@ $@ \mathrm{SiO}_{2}(\mathrm{MB})$,

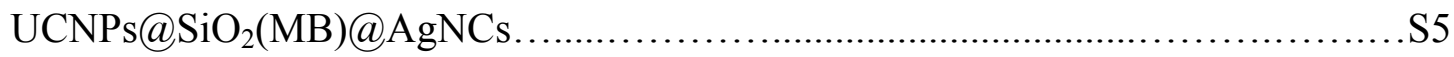

Figure S2 FT-IR spectra of UCNPs, UCNPs@ $\mathrm{SiO}_{2}$ and UCNPs@SiO $\mathrm{S}_{2}(\mathrm{MB}) \ldots \ldots . . . . . \mathrm{S} 6$

Figure S3 The photographs of UCNPs@ $\mathrm{SiO}_{2}(\mathrm{MB})\left(1 \mathrm{mg} \mathrm{mL}^{-1}\right)$ and MB $\left(1 \mathrm{mg} \mathrm{mL}^{-1}\right)$

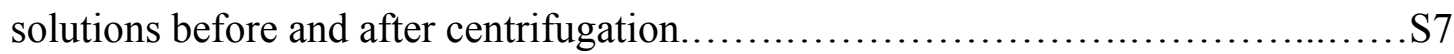

Figure S4 SEM image, EDS analysis and elemental mapping for

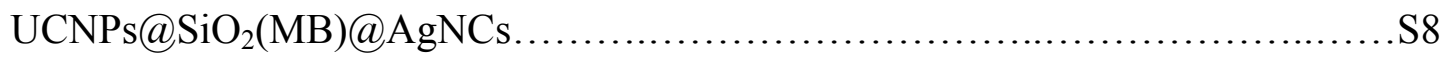

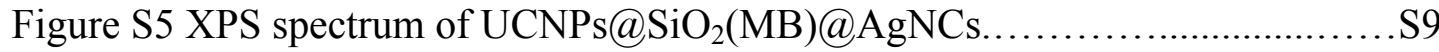

Figure S6 Efficiency of singlet oxygen $\left({ }^{1} \mathrm{O}_{2}\right)$ production by UCNPs@ $@ \mathrm{SiO}_{2}(\mathrm{MB})$, UCNPs@SiO $\mathrm{SiO}_{2}(\mathrm{MB}) @ \mathrm{AgNCs}$ upon 980 nm NIR irradiation..........................S10

Figure S7 The calibration graph between ICP-MS signal (cps) and the concentration of $\mathrm{Ag}^{+}$ S11

Figure S8. The survival rates of E. coli and S. aureus $\left(10^{6} \mathrm{cfu} \mathrm{mL}^{-1}\right)$ after incubation with NIR irradiation $\left(980 \mathrm{~nm}, 1 \mathrm{~W} \mathrm{~cm}^{-1}\right)$ for different time.

Table S1 The mass fraction of silver ions in $250 \mu \mathrm{g} \cdot \mathrm{L}^{-1} \mathrm{UCNPs} @ \mathrm{SiO}_{2}(\mathrm{MB}) @ \mathrm{AgNCs}$ as measured by ICP-MS. 


\section{EXPERIMENTAL SECTION}

\section{Materials Chemicals}

Yttrium chloride hexahydrate $\left(\mathrm{YCl}_{3} \cdot 6 \mathrm{H}_{2} \mathrm{O}, 99.9 \%\right)$, ytterbium chloride hexahydrate $\left(\mathrm{YbCl}_{3} \cdot 6 \mathrm{H}_{2} \mathrm{O}, 99.9 \%\right)$, erbium chloride $\left(\mathrm{ErCl}_{3}, 99.9 \%\right)$ and 1,3-diphenylisobenzofuran (DPBF) were purchased from Sigma-Aldrich (St. Louis, MO, USA). Oleic acid (OA), manganese chloride $\left(\mathrm{MnCl}_{2}\right)$ and sodium fluoride $(\mathrm{NaF})$ were received from Aladdin Reagent (Shanghai, China). Cyclohexane, hexylalcohol, Triton X-100, methylene blue (MB), dimethyl sulfoxide (DMSO), sodium chloride, sodium hydroxide, ammonium hydroxide, tetraethyl orthosilicate (TEOS), silver nitrate $\left(\mathrm{AgNO}_{3}\right)$ and $\mathrm{N}$ (3-triethoxysilylpropyl)ethylenediamine (TSD) were obtained from Sinopharm Chemical Reagent Co., Ltd. (Shanghai, China). Agar powder, ethanol and methyl alcohol were the product of Kemiou Chemical Reagent Co., Ltd. (Tianjin, China). Yeast extract and agar powder was received from Aoboxing Bio-Tech Co. (Beijing, China). Tryptone was acquired from Oxoid Ltd. (Hants, UK). DMEM high glucose medium, fetal bovine serum, penicillin, streptomycin and $0.25 \%$ trypsin-EDTA were achieved from Hyclone (Thermo Scientific, USA). 3-(4, 5-dimethylthiazol-2-yl)-2, 5diphenyltetrazolium bromide (MTT) assay kit was acquired from KeyGEN Biotech Co. (Nanjing, China). E. coli and S. aureus were provided by China Center of Industrial Culture Collection (Beijing, China). All reagents were of analytical reagent grade and used without further purification unless otherwise specified. Deionized water of $18 \mathrm{M} \Omega \mathrm{cm}^{-1}$ was sterilized and used throughout the experiments.

\section{Instrumentation}

Fluorescence spectra were recorded on an F-7000 fluorescence spectrophotometer (Hitachi, Japan) attached with an external $980 \mathrm{~nm}$ semiconductor laser (Changchun New Industries Optoelectronics Technology Co., Ltd., China). UV-vis absorption spectra were recorded on a U-3900 UV-vis spectrophotometer (Hitachi, Japan) with a 1.0-cm quartz cuvette at room temperature. X-ray diffraction patterns were recorded by empyrean series II X-ray diffraction spectrometer (PANanytical, Holland) by using $\mathrm{Cu}$ 
$\mathrm{K} \alpha$ radiation. Infrared spectra were obtained on a Nicolet-6700 FT-IR spectrophotometer (Thermo Instruments Inc., USA) using a KBr disk from 400-4000 $\mathrm{cm}^{-1}$ with a resolution of $1 \mathrm{~cm}^{-1}$. Transmission electron microscopy (TEM) images were recorded on a JEM-2100 (HR) transmission electron microscopy (JEOL, Japan). Scanning electronic microscopy (SEM) images were recorded using a SU 8010 scanning electron microscope (Hitachi High Technologies, Japan). The content of silver on the surface of silica was analyzed by EXPEC 7000 ICP-MS (Focused Photonics Inc., China). XPS spectra were analyzed by Thermo ESCALA 250Xi spectrometer electron spectroscopy (Thermo Instruments Inc., USA). Methylthiazolydiphenyl-tetrazolium bromide (MTT) assay was measured using a Synergy H1 ELISA plate reader at $490 \mathrm{~nm}$ (BioTek, USA). 


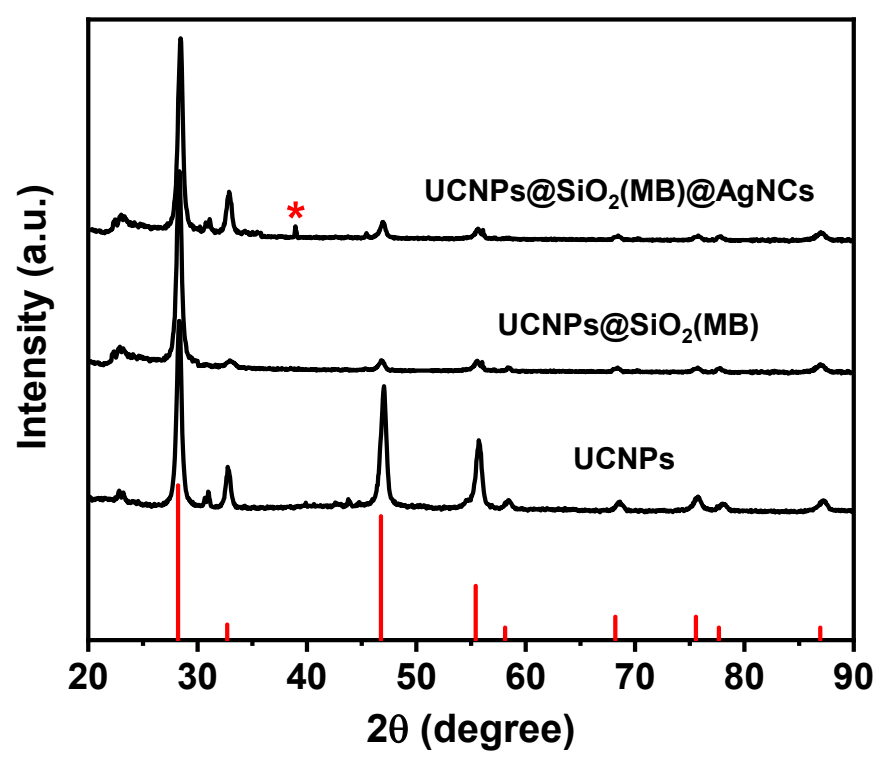

Figure S1. X-ray powder diffraction pattern of UCNPs, UCNPs@SiO $(\mathrm{MB})$, UCNPs@SiO $2(\mathrm{MB}) @ A g N C s$ and JCPDS card No. 77-2042 (red line). 


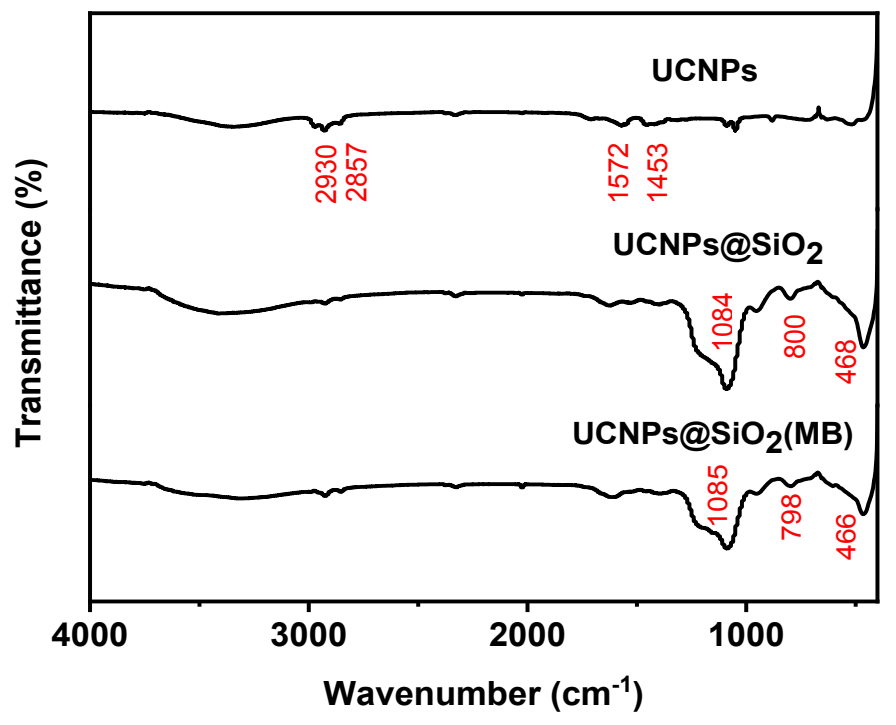

Figure S2. FT-IR spectra of UCNPs, UCNPs@SiO ${ }_{2}$ and UCNPs@ $@ \mathrm{SiO}_{2}(\mathrm{MB})$. 


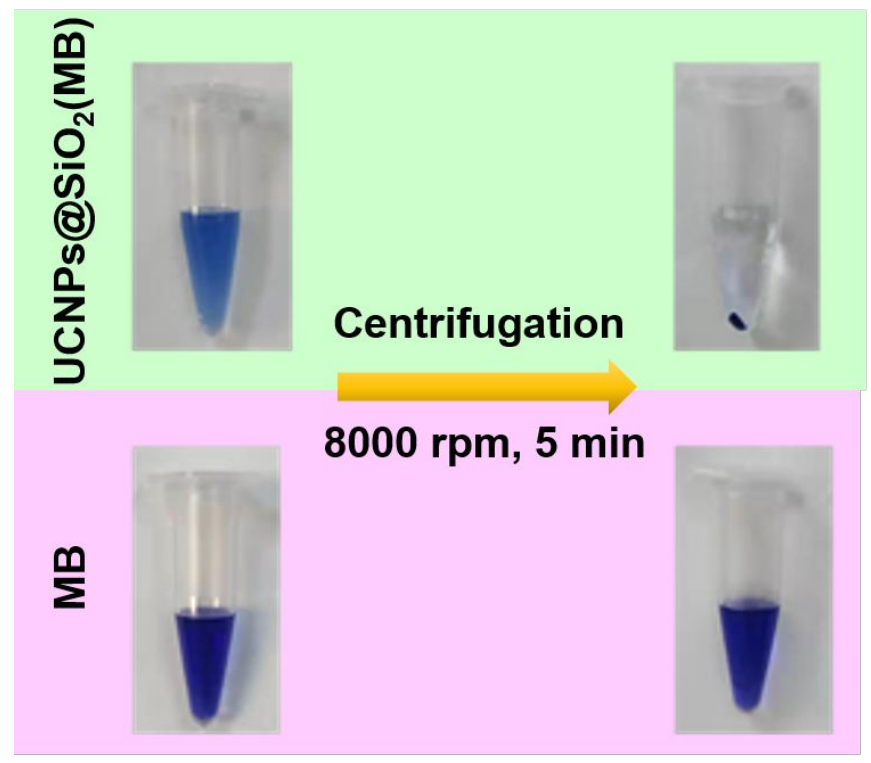

Figure S3. The comparison of UCNPs@SiO $2(\mathrm{MB})\left(1 \mathrm{mg} \mathrm{mL}^{-1}\right)$ and $\mathrm{MB}\left(1 \mathrm{mg} \mathrm{mL}^{-1}\right)$ solutions before and after centrifugation. 


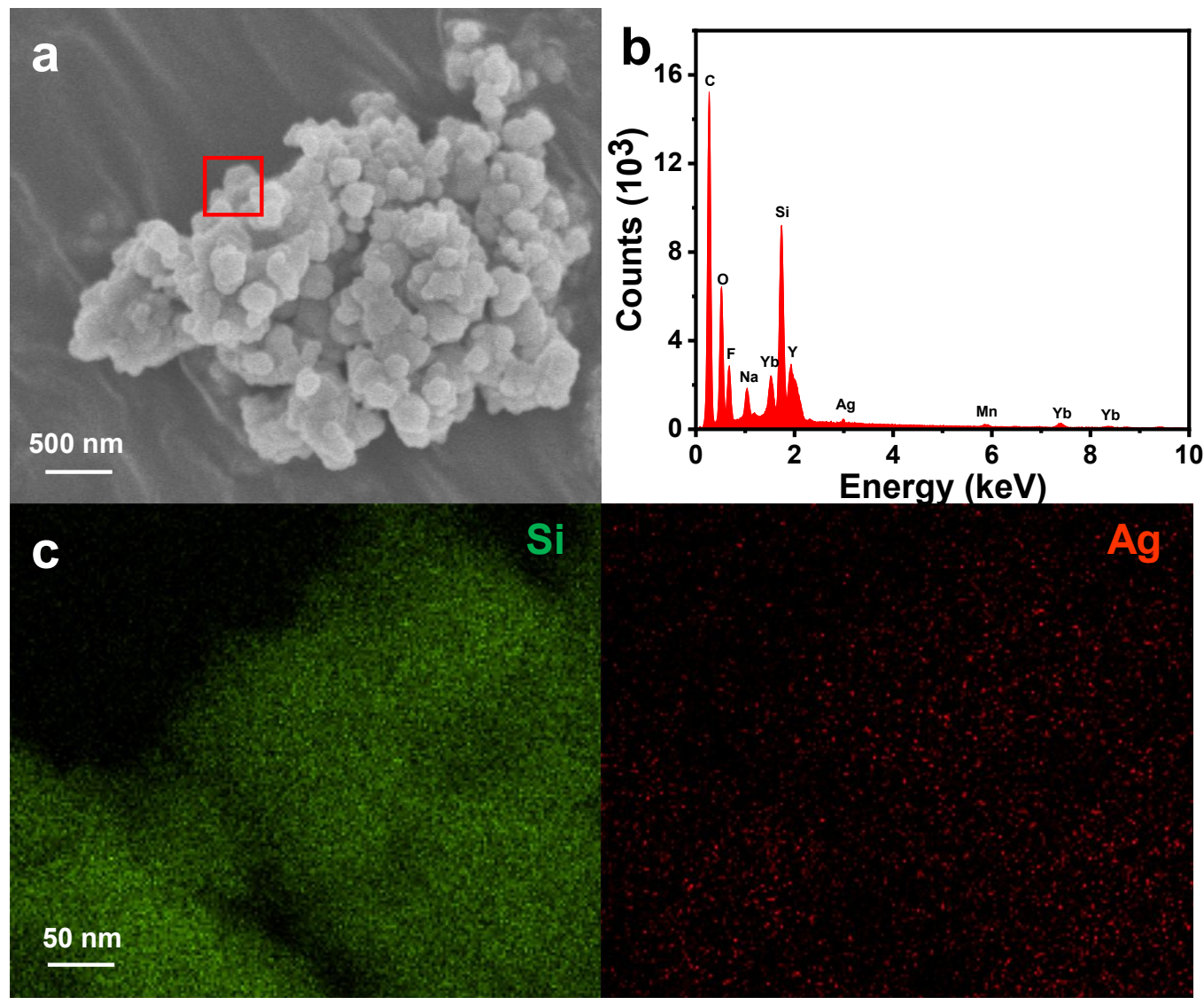

Figure S4. (a) SEM image for UCNPs@SiO $2(\mathrm{MB}) @ A g N C s .(b)$ EDS analysis of UCNPs@SiO $2(\mathrm{MB}) @ A g N C s$. (c) Elemental mapping of Si and Ag corresponding to the marked area in (a). 


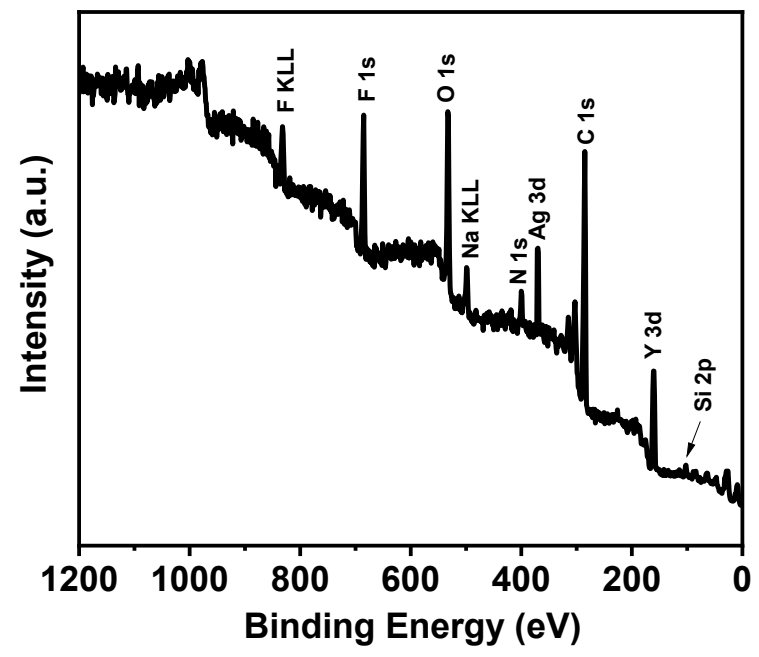

Figure S5. XPS spectrum of UCNPs@SiO $2(\mathrm{MB}) @ A g N C s$. 


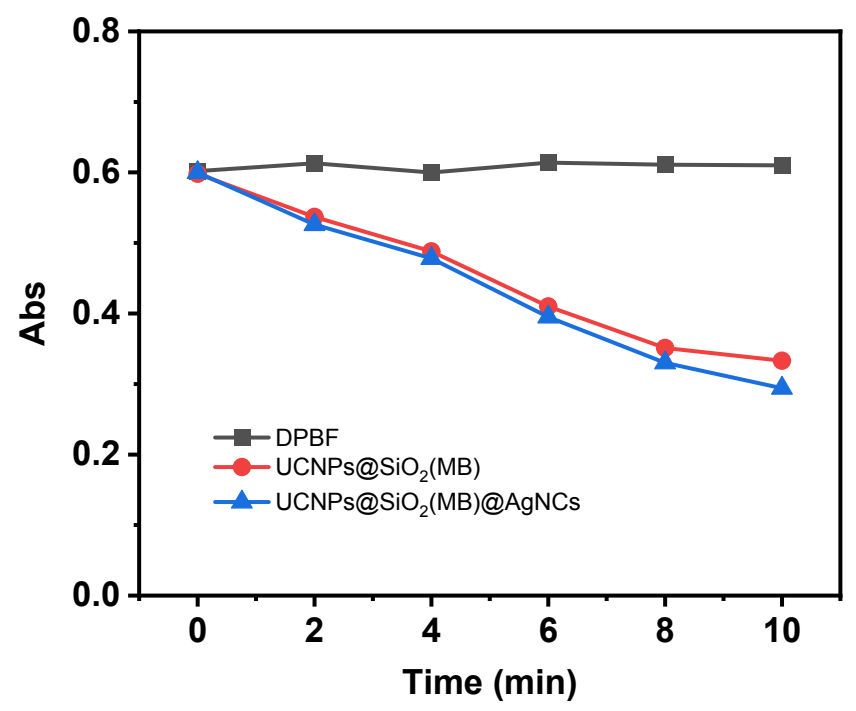

Figure S6. Efficiency of singlet oxygen $\left({ }^{1} \mathrm{O}_{2}\right)$ production by $\mathrm{UCNPs} @ \mathrm{SiO}_{2}(\mathrm{MB})$, UCNPs@SiO $2(\mathrm{MB}) @ A g N C s$ upon 980 nm NIR irradiation at a power density of 1.0 $\mathrm{W} \cdot \mathrm{cm}^{-2}$. The absorbance at $417 \mathrm{~nm}$ is recorded at the concentrations of $1.25,8.33$ and $8.33 \mu \mathrm{g} \mathrm{mL} \mathrm{m}^{-1}$ for DPBF, UCNPs@SiO $2(\mathrm{MB})$ and UCNPs@SiO $2(\mathrm{MB}) @ \mathrm{AgNCs}$, respectively. 


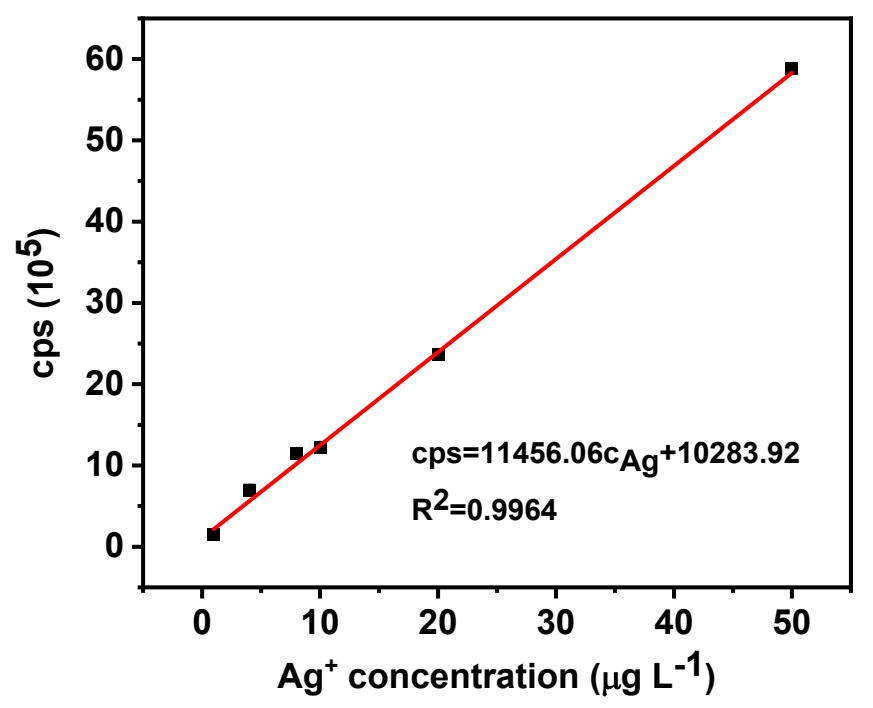

Figure S7. The calibration graph between ICP-MS signal (cps) and the concentration of $\mathrm{Ag}^{+}$within a range of $1-50 \mu \mathrm{g} \mathrm{L}^{-1}$. 


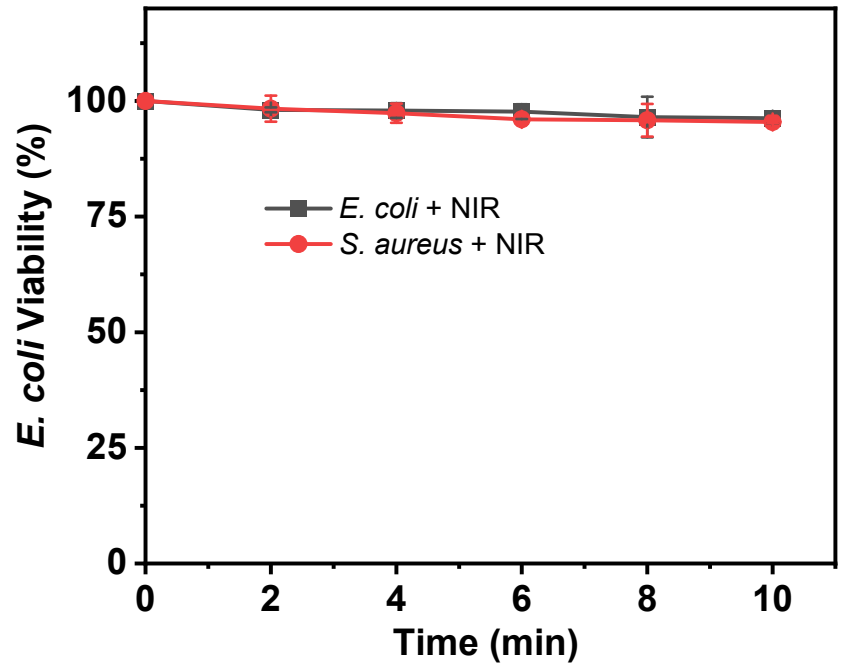

Figure S8. The survival rates of E. coli and $S$. aureus $\left(10^{6} \mathrm{cfu} \mathrm{mL}^{-1}\right)$ after incubation with NIR irradiation $\left(980 \mathrm{~nm}, 1 \mathrm{~W} \mathrm{~cm}^{-1}\right)$ for different time. 
Table S1. The mass fraction of silver ions in $250 \mu \mathrm{g} \cdot \mathrm{L}^{-1} \mathrm{UCNPs} @ \mathrm{SiO}_{2}(\mathrm{MB}) @ \mathrm{AgNCs}$ as measured by ICP-MS.

\begin{tabular}{llll}
\hline $\mathrm{Ag}^{+} \mathrm{cps}$ & $\mathrm{RSD}(\%)$ & $\mathrm{c}_{\mathrm{Ag}}(\mu \mathrm{g} \mathrm{L}-1)$ & Mass fraction of $\mathrm{Ag}^{+}(\%)$ \\
\hline $38528 \pm 1001.5$ & 2.8 & $2.42 \pm 0.22$ & $0.97 \pm 0.09$ \\
\hline
\end{tabular}

